\title{
Double-pulsed diffusional kurtosis imaging for the in vivo assessment of human brain microstructure
}

\author{
Edward S. Hui ${ }^{1 *}$ and Jens H. Jensen ${ }^{2,3}$ \\ ${ }^{1}$ Department of Diagnostic Radiology, Li Ka Shing Faculty of Medicine, The University of Hong Kong, \\ Pokfulam, Hong Kong SAR, China. \\ ${ }^{2}$ Department of Radiology and Radiological Science, ${ }^{3}$ Center for Biomedical Imaging, Medical University of \\ South Carolina, Charleston, South Carolina, USA. \\ * Corresponding Author: \\ Edward S. Hui, Ph.D. \\ Department of Diagnostic Radiology, Li Ka Shing Faculty of Medicine \\ The University of Hong Kong \\ LG3, Jockey Club Building for Interdisciplinary Research \\ 5 Sassoon Road, Pokfulam, Hong Kong \\ Tel: $+(852) 28315012$ \\ Email: edward.s.hui@gmail.com
}

Running Head: DP-DKI for the in vivo assessment of human brain microstructure

Word Count: 5694

Figures: 8 


\begin{abstract}
We have recently extended conventional single-pulsed-field-gradient (s-PFG) diffusional kurtosis imaging (DKI) to double-pulsed-field-gradient (d-PFG) diffusion MRI sequences, with a method known as double-pulsed DKI (DP-DKI). By virtue of a six-dimensional (6D) formulation for q-space, many of the results and insights of s-PFG DKI are generalized to those of DP-DKI. Owing to the fact that DP-DKI isolates the second order contributions to the d-PFG signal (i.e. second order in b-value), the 6D diffusional kurtosis encodes information beyond what is available from s-PFG sequences. Previously, we have demonstrated DPDKI for in vivo mouse brain at 7T, and it is the objective of this study to demonstrate the feasibility of DPDKI at $3 \mathrm{~T}$ for the in vivo assessment of human brain microstructure. In addition, an example is given of how to utilize the additional information obtained from DP-DKI for the purpose of biophysical modeling. The relationship between a specific microscopic anisotropy metric estimated from DP-DKI and other recently proposed measures is also discussed.
\end{abstract}

Keywords: double-pulsed-field-gradient, multiple-wave-vector, microscopic anisotropy, non-Gaussian diffusion, kurtosis, human 


\section{Introduction}

Diffusion tensor imaging (DTI) has become an invaluable tool for the in vivo assessment of neuroarchitecture. Of all DTI metrics, fractional anisotropy (FA) has been the most widely used biomarker for the characterization of white matter (WM) change in various neurological disorders. A premise of the FA is that it reflects the macroscopic anisotropy of water diffusion in WM that arises as a result of the coherent alignment of axonal fiber bundles. An alteration in fiber alignment or integrity, as in disease progression, can thus modulate the FA. However, because less than $10 \%$ of axonal fiber bundles are highly coherent, assessment of WM architecture using FA becomes ambiguous in a majority of WM voxels (De Santis et al., 2014). The classic case in point is the lower FA observed in regions with complex WM architecture, such as crossing or fanning fibers (Alexander et al., 2001). Moreover, FA is of limited utility in gray matter (GM), as GM has a low macroscopic anisotropy. This is in spite of GM having substantial microscopic diffusion anisotropy arising from water restricted by neurites (i.e., axons and dendrites) (Shemesh et al., 2010b). Improved means for quantifying the diffusion anisotropy of brain tissue with complex fiber geometries may potentially provide more sensitive and specific biomarkers of microstructural changes due to pathophysiological processes.

Mitra (Mitra, 1995) considered the application of pulsed-field-gradient (PFG) NMR with multiple wave vector diffusion encodings to the quantification of the diffusion dynamics for spins within porous media. The extension of this method to MRI is known variously as multiple-PFG, multiple-wave-vector, or multiplepulsed diffusion MRI. The case with two diffusion-encoding wave vectors (i.e., double-pulsed diffusion MRI) (Shemesh et al., 2010b) is the simplest and most commonly utilized variant. Double-pulsed diffusion MRI has been applied by several groups as a means of assessing diffusion anisotropy (Callaghan and Komlosh, 2002; Jensen et al., 2014; Jespersen et al., 2013; Komlosh et al., 2008, 2007; Lasič et al., 2014; Lawrenz and Finsterbusch, 2014, 2013, 2011; Lawrenz et al., 2010; Ozarslan and Basser, 2008; Ozarslan, 2009; Shemesh and Cohen, 2011a; Shemesh et al., 2012a, 2012b; Szczepankiewicz et al., 2014).

In a seminal paper by Jespersen (Jespersen, 2012), it is shown that the information obtained with double-pulsed diffusion MRI and with conventional s-PFG experiments are equivalent at low diffusion weightings, although for higher diffusion weightings new information is accessible with d-PFG sequences. This follows from the fact that the standard diffusion tensor, as obtained with DTI, is sufficient for fully characterizing the diffusion MRI signal to leading order in the diffusion weighting for arbitrary pulse sequences (Jensen, 2014). In this low diffusion-weighting limit, the Gaussian approximation of DTI is all that is needed, since non-Gaussian diffusion effects are small. Thus for double-pulsed diffusion MRI to yield truly novel information, beyond what is provided by s-PFG methods, higher diffusion weightings must be applied so that non-Gaussian diffusion effects become substantial. 
In order to more conveniently quantify the leading non-Gaussian diffusion effects for double-pulsed diffusion MRI, we have recently introduced DP-DKI, which employs d-PFG sequences (Jensen et al., 2014). As a part of the DP-DKI formalism, the two three-dimensional (3D) diffusion wave vectors are interpreted as a single 6D wave vector. In this way, many of the concepts and formulae from conventional DKI may be generalized to DP-DKI in a straightforward manner. In particular, DP-DKI is able to compactly quantify all contributions to the d-PFG signal magnitude up to second order in the b-value. These second order contributions comprise the novel information that is, in practice, most easily measurable with double-pulsed diffusion MRI.

This novel information is closely linked to the notion of microscopic diffusion anisotropy. Most prior studies have conceived of microscopic anisotropy as encompassing macroscopic anisotropy, so that any medium with a nonzero FA would be regarded as possessing both macroscopic and microscopic anisotropy (Lasič et al., 2014; Shemesh et al., 2010b). We shall refer to this as "type I" microscopic anisotropy. Alternatively, one can define "type II" microscopic anisotropy as being diffusion anisotropy that can only be detected with multiple-PFG sequences. In this way, a medium (e.g., a single domain liquid crystal) could be macroscopically anisotropic without necessarily having type II microscopic anisotropy. While these two conceptions of microscopic anisotropy are closely related, type II microscopic anisotropy arises naturally out of the DP-DKI approach. For systems without macroscopic anisotropy, type I and type II microscopic anisotropy are equivalent and may both be nonzero. For example, water confined to an ensemble of many randomly oriented cylindrical pores is macroscopically isotropic (Shemesh et al., 2010a), even though the diffusion within each pore is highly anisotropic. This is called microscopic anisotropy, since measures that only quantify the anisotropy of the full ensemble are insensitive to this property.

Several related metrics have been proposed for quantifying type I microscopic anisotropy with doublepulsed diffusion MRI data. Lawrenz and coworkers (Lawrenz et al., 2010) derived a rotationally invariant metric of microscopic anisotropy for porous media, known as $\mathrm{I}_{\mathrm{MA}}$, from the Taylor series of the d-PFG signal in the long diffusion time limit. Shemesh and coworkers (Shemesh et al., 2012a) also proposed a metric of microscopic anisotropy, known as apparent eccentricity index, which is derived from the dependence of dPFG signal on the angle $\theta$ between the diffusion wave vector for the first and second blocks of PFG. Subsequently, Jespersen and coworkers (Jespersen et al., 2014, 2013) introduced an apparent compartmental eccentricity that may be applied for all diffusion times and is proportional to $\mathrm{I}_{\mathrm{MA}}$, in the long diffusion time limit. They also defined a fractional eccentricity (FE) that recasts the same information in manner more analogous to the FA, so that the FE equals the FA whenever the type II microscopic anisotropy vanishes. With magic-angle-spinning diffusion MRI, one can also measure a "microscopic fractional anisotropy" $(\mu \mathrm{FA})$ (Lasič et al., 2014; Szczepankiewicz et al., 2014). It is argued by Jespersen and coworkers that FE $=\mu F A$, although the exact equality depends on corrections included in an erratum (Jespersen et al., 2014). 
In our previous work (Jensen et al., 2014), we have defined a general rotational invariant for DP-DKI, $\widetilde{W}$, that is equal to the mean of the 6D diffusional kurtosis tensor averaged over all possible 6D directions. In addition, we have shown that, for multiple Gaussian compartment (MGC) models, $\widetilde{W}$ provides information closely related to type II microscopic anistropy. In this paper, we consider the relationship of $\widetilde{W}$ to some of the indices of microscopic anisotropy discussed above.

We have previously described the DP-DKI formalism in detail and given preliminary results for mouse brain. Here our primary aim is to demonstrate the feasibility of the method for in vivo human imaging. Beyond this, we also expand upon the physical meaning of the diffusion metric $\widetilde{W}$ and give an example of its application to tissue modeling of gray matter.

\section{Methods}

\section{Theory}

In this section, we will briefly describe the theory underlying DP-DKI; please refer to (Jensen et al., 2014) for a more detailed description thereof.

An example d-PFG MRI sequence consists of two blocks of diffusion gradients, one between the $90^{\circ}$ excitation and $180^{\circ}$ refocusing pulses, and the other after the $180^{\circ}$ refocusing pulse (see Figure 1). The pulse duration $\delta$ and diffusion time $\Delta$ for the two diffusion gradient blocks are assumed to be identical, and the interval between the end of the first and the beginning of the second block is characterized by a mixing time $\tau$. The gradient strengths for the first and second blocks are $g$ and $g^{\prime}$, respectively, and their corresponding bvalues are defined as $b \equiv(\gamma \delta g)^{2}(\Delta-\delta / 3)$ and $b^{\prime} \equiv\left(\gamma \delta g^{\prime}\right)^{2}(\Delta-\delta / 3)$, where $\gamma$ is the proton gyromagnetic ratio. The $3 \mathrm{D}$ diffusion wave vectors for the first and second blocks are $\mathbf{q} \equiv \gamma \delta g \mathbf{n} / 2 \pi$ and $\mathbf{q}^{\prime} \equiv \gamma \delta g^{\prime} \mathbf{n}^{\prime} / 2 \pi$, respectively. Here $\mathbf{n}$ and $\mathbf{n}^{\prime}$ are the standard 3D unit vectors associated with the corresponding diffusion wave vector.

The pair of diffusion wave vectors $\left(\mathbf{q}, \mathbf{q}^{\prime}\right)$ can be regarded as a single $6 \mathrm{D}$ diffusion wave vector $\widetilde{\mathbf{q}} \equiv\left(\mathbf{q}, \mathbf{q}^{\prime}\right)$, whereby the first three components of $\widetilde{\mathbf{q}}$ correspond to $\mathbf{q}$ and the last three components of $\widetilde{\mathbf{q}}$ correspond to $\mathbf{q}^{\prime}$. The $6 \mathrm{D}$ unit vector associated with the direction of $\widetilde{\mathbf{q}}$ is $\widetilde{\mathbf{n}} \equiv \widetilde{\mathbf{q}} / \tilde{q}$, where $\widetilde{q} \equiv|\widetilde{\mathbf{q}}|$. The $6 \mathrm{D}$ bvalue of a d-PFG sequence is then given by $\tilde{b} \equiv(2 \pi \tilde{q})^{2}(\Delta-\delta / 3)=b+b^{\prime}$. The logarithm of the d-PFG signal magnitude $\tilde{S}(\tilde{b}, \widetilde{\mathbf{n}})$ can be expanded in powers of $\tilde{b}$ as

$$
\ln \frac{\tilde{S}(\tilde{b}, \widetilde{\mathbf{n}})}{\tilde{S}_{0}}=-\tilde{b} \widetilde{D}(\widetilde{\mathbf{n}})+\frac{1}{6} \widetilde{b}^{2} \widetilde{D}(\widetilde{\mathbf{n}})^{2} \widetilde{K}(\widetilde{\mathbf{n}})+O\left(\tilde{b}^{3}\right),
$$


where $\widetilde{D}(\widetilde{\mathbf{n}})$ and $\widetilde{K}(\widetilde{\mathbf{n}})$ are the $6 \mathrm{D}$ directional diffusivity and diffusional kurtosis, respectively, and $\tilde{S}_{0} \equiv$ $\tilde{S}(0, \widetilde{\mathbf{n}})$ is the d-PFG signal magnitude without diffusion weighting. Note that a tilde is used to indicate $6 \mathrm{D}$ quantities.

The relationships between the $6 \mathrm{D}$ diffusion tensor $\widetilde{\mathbf{D}}$ and diffusional kurtosis tensor $\widetilde{\mathbf{W}}$, and the corresponding 6D directional diffusivity and diffusional kurtosis are

$$
\widetilde{D}(\widetilde{\mathbf{n}})=\sum_{\alpha, \beta=1}^{6} \tilde{n}_{\alpha} \tilde{n}_{\beta} \widetilde{D}_{\alpha \beta},
$$

and

$$
\widetilde{K}(\widetilde{\mathbf{n}})=\left(\frac{\widetilde{D}}{\widetilde{D}(\widetilde{\mathbf{n}})}\right)^{2} \sum_{\alpha, \beta, \gamma, \delta=1}^{6} \tilde{n}_{\alpha} \tilde{n}_{\beta} \tilde{n}_{\gamma} \tilde{n}_{\delta} \widetilde{W}_{\alpha \beta \gamma \delta}
$$

where $\tilde{n}_{\alpha}, \widetilde{D}_{\alpha \beta}$ and $\widetilde{W}_{\alpha \beta \gamma \delta}$ are components of $\widetilde{\mathbf{n}}, \widetilde{\mathbf{D}}$ and $\widetilde{\mathbf{W}}$ respectively, and $\widetilde{\bar{D}}=\operatorname{tr}(\widetilde{\mathbf{D}}) / 6$ is the $6 \mathrm{D}$ mean diffusivity (MD). While $\widetilde{\mathbf{D}}$ has a total of 36 components, only 12 of these are independent, as a consequence of symmetry and time reversal invariance. Similarly, $\widetilde{\mathbf{W}}$ has only 66 independent components out of a total of 1296. This may be compared to the 6 independent components for the conventional 3D diffusion tensor $\mathbf{D}$ and to the 15 independent components for the 3D kurtosis tensor $\mathbf{W}$.

As shown by (Jespersen, 2012), $\widetilde{\mathbf{D}}$ may be constructed from the conventional 3D diffusion tensor $\mathbf{D}$ according to

$$
\widetilde{\mathbf{D}}(\Delta, \tau)=\left[\begin{array}{cc}
\mathbf{D}(\Delta) & \mathbf{C}(\Delta, \tau) \\
\mathbf{C}(\Delta, \tau) & \mathbf{D}(\Delta)
\end{array}\right]
$$

where

$$
\mathbf{C}(\Delta, \tau) \equiv \frac{1}{2 \Delta}[(2 \Delta+\tau) \mathbf{D}(2 \Delta+\tau)+\tau \mathbf{D}(\tau)-2(\Delta+\tau) \mathbf{D}(\Delta+\tau)]
$$

As a consequence, $\widetilde{\mathbf{D}}$ could be estimated from s-PFG experiments that are performed at three different diffusion times. Following from Eq. [4], $\widetilde{\mathbf{D}}$ and $\mathbf{D}$ have the same $\mathrm{MD}$, i.e. $\widetilde{\bar{D}}=\operatorname{tr}(\mathbf{D}) / 3=\bar{D}$. Hence of the two $6 \mathrm{D}$ tensors, only $\widetilde{\mathbf{W}}$ contains unique information beyond that available from s-PFG measurements.

\section{Experiment}

To achieve an efficient in vivo DP-DKI protocol, the mean of $\widetilde{\mathbf{W}}$ will be estimated in lieu of the full $\widetilde{\mathbf{W}}$, for which a minimum of 66 independent diffusion-encoding directions would be required. As shown by Jensen and coworkers (Jensen et al., 2014), the mean of $\widetilde{\mathbf{W}}$ may be calculated from

$$
\begin{aligned}
& \widetilde{\bar{W}}=\frac{1}{8}\left(\widetilde{W}_{1111}+\widetilde{W}_{2222}+\widetilde{W}_{3333}+2 \widetilde{W}_{1122}+2 \widetilde{W}_{1133}+2 \widetilde{W}_{2233}\right. \\
& \left.+\widetilde{W}_{1144}+\widetilde{W}_{2255}+\widetilde{W}_{3366}+2 \widetilde{W}_{1155}+2 \widetilde{W}_{1166}+2 \widetilde{W}_{2266}\right) .
\end{aligned}
$$

On the other hand, the mean of the standard 3D diffusional kurtosis tensor $\mathbf{W}$, which is strongly correlated with and approximately equal to the conventional mean kurtosis, is given by (Hansen et al., 2014, 2013)

$$
\bar{W}=\frac{1}{5}\left(W_{1111}+W_{2222}+W_{3333}+2 W_{1122}+2 W_{1133}+2 W_{2233}\right)
$$


where $W_{i j k l}$ is a component of $\mathbf{W}$. One may show that 21 and 9 diffusion directions are sufficient for the estimation of $\widetilde{W}$ and $\bar{W}$, respectively. Because 9 of the 21 directions needed for $\widetilde{W}$ are the same as those needed for $\bar{W}$, a single diffusion-encoding scheme of 21 directions (see Table 1) is in fact adequate for the estimation of both quantities. The difference $\bar{W}-\widetilde{W} \equiv \delta \bar{W}$ has been proposed as an index of type II microscopic anisotropy and should be nonnegative for any MGC model (Jensen et al., 2014).

The following quantities are constructed for estimating $\widetilde{W}$ and $\bar{W}$ from d-PFG signals

$$
\tilde{\psi}(\tilde{b})=\frac{1}{12} \sum_{m=4}^{15} \ln \tilde{S}\left(\tilde{b}, \widetilde{\mathbf{n}}_{m}\right)+\frac{1}{24} \sum_{m=16}^{21} \ln \tilde{S}\left(\tilde{b}, \widetilde{\mathbf{n}}_{m}\right)-\frac{1}{12} \sum_{m=1}^{3} \ln \tilde{S}\left(\tilde{b}, \widetilde{\mathbf{n}}_{m}\right)
$$

and

$$
\psi(\tilde{b})=\frac{1}{15} \sum_{m=1}^{3} \ln \tilde{S}\left(\tilde{b}, \widetilde{\mathbf{n}}_{m}\right)+\frac{2}{15} \sum_{m=4}^{9} \ln \tilde{S}\left(\tilde{b}, \widetilde{\mathbf{n}}_{m}\right),
$$

respectively, where $\widetilde{\mathbf{n}}_{m}$ is the set of $216 \mathrm{D}$ diffusion-encoding directions listed in Table 1. Expanding these equations in powers of $\tilde{b}$ yields (Jensen et al., 2014)

$$
\tilde{\psi}(\tilde{b})=\ln \tilde{S}_{0}-\tilde{b} \widetilde{\bar{D}}+\frac{1}{6} \tilde{b}^{2} \widetilde{D}^{2} \widetilde{\bar{W}}+O\left(\tilde{b}^{3}\right),
$$

and (Hansen et al., 2014, 2013)

$$
\psi(\tilde{b})=\ln \tilde{S}_{0}-\tilde{b} \bar{D}+\frac{1}{6} \tilde{b}^{2} \bar{D}^{2} \bar{W}+O\left(\tilde{b}^{3}\right) .
$$

These results indicate that $\widetilde{\bar{D}}$ and $\widetilde{W}$ may be estimated from a fit to Eq. [10] for $\tilde{\psi}(\tilde{b})$, whilst $\bar{D}$ and $\bar{W}$ can be determined from a fit to Eq. [11] for $\psi(\tilde{b})$. In carrying out these fits, the $O\left(\tilde{b}^{3}\right)$ terms in Eqs. [10] and [11] are neglected.

Experiments were performed on a 3.0 T whole-body MR scanner (Achieva TX, Philips Healthcare, Best, The Netherlands) equipped with a Quasar Dual gradient system (gradient amplitudes up to $80 \mathrm{mT} / \mathrm{m}$ ) using an 8-channel receive-only head coil. Four healthy volunteers were recruited with informed consent. An in-house spin-echo echo-planar-imaging sequence with d-PFG was used (Figure 1). Diffusion-weighted images (DWIs) were acquired with 5 6D b-values (0, 500, 1000, 1500, $\left.2000 \mathrm{~s} / \mathrm{mm}^{2}\right)$ along 21 diffusionencoding directions (Table 1) in 15 slices with the following imaging parameters: $\delta / \Delta / \tau=14.9 / 21.4 / 34.1 \mathrm{~ms}$, $\mathrm{TR} / \mathrm{TE}=5500 / 112 \mathrm{~ms}$, field-of-view $=230 \times 230 \mathrm{~mm}^{2}$, acquisition matrix $=76 \times 76$, SENSE factor of 2 along the phase encoding direction (anterior-posterior), slice thickness $=5 \mathrm{~mm}$ (no gap) and receiver bandwidth $=3657 \mathrm{~Hz} /$ pixel. Each experiment was performed twice for signal averaging, except for the $\tilde{b}=0$ measurement, which was performed 17 times, resulting in a total acquisition time of approximately 19 minutes.

Spatial smoothing using a 3D Gaussian filter (with a full-width-at-half-maximum of $3.75 \mathrm{~mm}$ ) was applied to all DP-DKI data before fitting. Linear least square estimations of $\widetilde{\bar{D}}$ and $\widetilde{W}$ (Eq. [10]) as well as $\bar{D}$ and $\bar{W}$ (Eq. [11]) were performed on a voxel-by-voxel basis for all filtered DP-DKI data. Owing to the zero diffusion weighting of the second block of diffusion gradients $\left(b^{\prime}=0\right)$ for the first 9 diffusion-encoding 
directions, the corresponding DP-DKI data were regarded as conventional s-PFG measurements. Conventional s-PFG diffusion metrics, such as fractional anisotropy (FA), were thus subsequently estimated. Region-ofinterests (ROIs) covering the splenium of the corpus callosum $(\mathrm{CC}$, mean number of voxels $=58$ ), corticospinal tract $(\mathrm{CT}$, mean number of voxels $=30)$, putamen $(\mathrm{PU}$, mean number of voxels $=86)$, thalamus (THA, mean number of voxels $=84$ ) and caudate nucleus (CAU, mean number of voxels $=26$ ) were manually selected (see the insets in Figure 2 for ROI definitions). Cerebral spinal fluid (CSF) was removed by using a threshold of $\mathrm{MD} \geq 1.2 \mu \mathrm{m}^{2} / \mathrm{ms}$. All measurements were obtained by taking the average of all voxels in the

corresponding ROIs. Paired t-tests were performed to compare $\widetilde{D}$ and $\widetilde{\bar{D}}$ as well as $\bar{W}$ and $\widetilde{W}$ for all ROIs and all subjects. Tests with p-value $<0.01$ were considered as statistically significant. All computations were performed using MATLAB (Mathworks, Natick, Massachusetts).

\section{Results}

Figure 2 shows the $\tilde{\psi}(\tilde{b})$ (square) and $\psi(\tilde{b})$ (circle) measured from the normalized d-PFG signal $\tilde{S}(\tilde{b}, \widetilde{\mathbf{n}}) / \tilde{S}_{0}$ in the CC, CT, THA, PU and CAU of a representative volunteer. The data points are expressed as the mean and standard deviation of all voxel values in the corresponding ROI. The quadratic fits to the $\tilde{\psi}$ (solid line) and $\psi$ (dashed line) are also shown, and the means and standard deviations of all fitted $\bar{D}, \widetilde{\bar{D}}, \bar{W}$ and $\widetilde{W}$ in each ROI are listed in Table 2. The ROI definitions for each brain structure are also shown in the inset of the corresponding signal plot. As expected, $\widetilde{\bar{D}}$ and $\bar{D}$ are almost identical. The values of $\delta \bar{W}$ for the WM structures (CC and CT) are the largest, followed by THA, and GM structures (PU and CAU).

The dependence of the d-PFG signal at b-value of $2000 \mathrm{~s} / \mathrm{mm}^{2}$ (expressed as percent change in signal variation) on the angle $\theta$ between the diffusion wave vector for the first and second blocks of PFG for all ROIs of a representative volunteer is shown in Figure 3. With reference to Table 1, the four different signal curves were obtained by combining the data with the first wave vector being fixed while the second vector varied. The angular dependence of d-PFG largely corroborates with the ROI analysis in Figure 2, with WM having the biggest signal modulations, and GM being the smallest.

The ROI measurements of $\bar{D}, \widetilde{D},|\bar{D}-\widetilde{D}|, \bar{W}, \widetilde{W}$ and $\delta \bar{W}$ for all subjects are shown in Figure 4. The measurements are expressed as mean and standard deviation of all voxel values in the corresponding ROI for each subject. Despite the absolute difference between $\bar{D}$ and $\widetilde{\bar{D}}$ being very small (maximum absolute difference of only $0.07 \pm 0.02 \mu \mathrm{m}^{2} / \mathrm{ms}$ ), there are statistically significant differences between the two metrics in several of the ROIs. The mean and standard deviation of $\delta \bar{W}$ for all subjects in CC, CT, THA, PU and CAU are $0.22 \pm 0.03,0.25 \pm 0.05,0.14 \pm 0.05,0.09 \pm 0.01$ and $0.04 \pm 0.01$, respectively. The differences between $\bar{W}$ and $\widetilde{W}$ were statistically significant for all ROIs and subjects except the CAU of subjects 1 to 3 . 
Representative $\bar{D}, \widetilde{D},|\bar{D}-\widetilde{\bar{D}}|, \bar{W}, \widetilde{W}$ and $\delta \bar{W}$ maps are displayed in Figure 5. FA maps computed from the DP-DKI data acquired with the first 9 diffusion-encoding directions are also shown for comparison. WM and deep GM regions have higher $\delta \bar{W}$ than cortical regions and ventricles. Notice that the contrast for $\delta \bar{W}$ in WM differs from that for FA. To illustrate inter-subject variability, the $\widetilde{W}$ and $\delta \bar{W}$ maps of all subjects are shown in Figure 6. Despite of the dark voids in $\widetilde{W}$, the maps are fairly consistent amongst the subjects.

Figure 7 shows the scatter plot for $\widetilde{W}$ (left) and $\delta \bar{W}$ (right) against $\bar{W}$ for various brain regions of all subjects. The Spearman correlation coefficient of $\widetilde{W}$ for WM structures is 0.72 , for THA is 0.75 , and for GM structures is 0.79 . Whereas that of $\delta W$ for WM structures is 0.27 , for THA is 0.48 , and for GM structures is 0.34. These results indicate that $\delta W$ carries substantial independent information as compared to $\bar{W}$.

\section{Discussion}

The major purpose of this study has been to demonstrate the feasibility of DP-DKI for in vivo imaging of human brain. A key to our approach has been to restrict the data acquisition to the $216 \mathrm{D}$ directions listed in Table 1. Although this does not allow for the calculation of the full 6D kurtosis tensor, it does suffice for

determining its mean, $\widetilde{W}$, as well as the mean of the 3D kurtosis tensor, $\bar{W}$. From the difference of these two quantities, one finds $\delta \bar{W}$, which is an index of type II microscopic anisotropy for MGC models. Another important aspect of our experiment has been the use of a scanner with $80 \mathrm{mT} / \mathrm{m}$ gradients, which permits a shorter echo time than is possible with scanners having the more typical $40 \mathrm{mT} / \mathrm{m}$ gradient sets. This shorter echo time both improves the signal-to-noise ratio (SNR) and reduces the total acquisition time. While the 19 min acquisition time of our proposed protocol may be acceptable for research studies, it is still too lengthy for most clinical applications. However, advanced acquisition methods such as simultaneous multi-slice imaging (Feinberg and Setsompop, 2013) offer the potential of substantially shorter acquisition times. In addition, refinements to our protocol, such as an optimized choice of b-values, may help to both reduce scan time and improve the quality of the parametric maps. It should be noted that the choice of the maximum b-value is critical for DP-DKI, since it should be large enough so that non-Gaussian diffusion effects are apparent but not so large as to invalidate the expansion of Eq. [1].

\section{Contrast mechanism of $\delta \bar{W}$}

Type II microscopic anisotropy, as indexed by the rotationally invariant DP-DKI metric $\delta \bar{W}$, reveals microstructural information that is different and complementary to that of conventional FA. As previously discussed by Jensen and coworkers (Jensen et al., 2014), the use of $\delta \bar{W}$ for the assessment of microscopic anisotropy is motivated by considering MGC models that assume no inter-compartmental water exchange. 
This class of models has been widely applied to analyze diffusion MRI data in brain (Panagiotaki et al., 2012). In the context of MGC models, the presence of type II microscopic anisotropy means that the difference between at least one pair of compartmental diffusion tensors is not an isotropic tensor (i.e, not simply proportional to the identity matrix) (Jensen et al., 2014). For such models, it can be shown that (see Appendix)

$$
\delta \bar{W}=\frac{9}{20 \bar{D}^{2}}\left(\delta^{2} \lambda_{c}-\delta^{2} \lambda\right)
$$

where $\delta^{2} \lambda_{c}$ is the mean variance of the compartmental 3D diffusion tensor eigenvalues and $\delta^{2} \lambda$ is the variance of the eigenvalues of the $3 \mathrm{D}$ diffusion tensor for the full system. In the absence of type II microscopic anisotropy, we have $\delta^{2} \lambda=\delta^{2} \lambda_{c}$ so that $\delta \bar{W}$ vanishes. A near zero value for $\delta \bar{W}$ may then be expected for tissue compartments where only free fluid exists, such as the ventricles. It is also possible to prove that (Jensen et al., 2014)

$$
\frac{5}{8} \bar{W} \leq \widetilde{W} \leq \bar{W}
$$

which gives an allowed range of $\widetilde{\bar{W}}$ for these models. When all the compartments have the same MD but different directional diffusivities, then $\widetilde{W}=5 \bar{W} / 8$. Broadly speaking, a larger $\delta \bar{W}$ indicates a higher degree of type II microscopic anisotropy.

However, as discussed in our prior work (Jensen et al., 2014), $\delta \bar{W}$ may, in practice, also be affected by factors such as inter-compartmental water exchange not included in simple MGC models (particularly for long mixing times), compartmental differences in T2 (Ackerman and Neil, 2010), poor SNR, or imaging artifacts. For our experiment, the mixing time of $34.1 \mathrm{~ms}$ is small in comparison to estimated water exchange times for white matter of about $1 \mathrm{~s}$ (Nilsson et al., 2013), and so water exchange likely had little effect for our $\delta \bar{W}$ values in white matter. For very short mixing times, it is possible that $\delta \bar{W}$ would be influenced by diffusion restrictions caused by the presence of intra-compartmental non-Gaussian diffusion effects. Additionally, it may be argued that $\delta \bar{W}$ only depends weakly on diffusion gradient pulse duration (Jensen and Helpern, 2011; Jensen et al., 2014).

The results of our study confirm that $\widetilde{D}=\bar{D}$, within experimental uncertainties (see Table 2), as expected theoretically. We also find that the inequality of Eq. [13] is obeyed in a majority of voxels (about $63 \%$ of the whole brain), as is required for all MGC models. As illustrated in Figure 5, $\delta \bar{W}$ exhibits a different pattern of contrast than the FA. In particular, $\delta \bar{W}$ is relatively elevated in some low FA regions, such as the thalamus. Of additional note is the cortical WM with high $\delta \bar{W}$ (for example, the posterior cortical WM regions in the third column of Figure 5). This contrast may arise from voxels that are composed of both WM and GM. In the cortical GM regions, the angular modulation of d-PFG signal appears small and $\delta \bar{W}$ appears low in comparison to WM. However, because our slice thickness of $5 \mathrm{~mm}$ is large relative to the thickness of the cortical ribbon, CSF partial volume effects are likely substantial, and consequently the significance of the observed low $\delta \bar{W}$ is unclear. It may also be possible that the current mixing time is too short to observe the substantial microscopic diffusion anisotropy as seen by Shemesh and coworkers (Shemesh et al., 2010b). It 
should be noted that our measured values for $\delta \bar{W}$ are similar to those in our prior animal study (Jensen et al., 2014).

\section{Biophysical modeling of brain microstructure using DP-DKI}

The additional information provided by DP-DKI can be utilized for the biophysical modeling of brain microstructure. As a simple example for modeling GM, consider a two-compartment model without water exchange. The model consists of an extra-neurite compartment with Gaussian diffusion and an intra-neurite compartment, which is assumed to be comprised of isotropically oriented neurites. The neurites are modeled as thin cylinders such that diffusion parallel to the long-axis of a cylinder is Gaussian and that perpendicular to the long-axis is zero. The diffusion dynamics is thus macroscopically isotropic in both compartments. Since the diffusion within each cylinder is Gaussian, both the intra-neurite compartment and the full system correspond to MGC models (albeit with an infinite number of Gaussian compartments comprising the intraneurite compartment). Hence Eq. [13] is applicable to both the intra-neurite compartment alone and to the full system. Because the neurites are oriented in different directions, the diffusion dynamics for the intra-neurite compartment taken as a whole is non-Gaussian with a kurtosis of $12 / 5$, even though the individual neurites have Gaussian diffusion (Fieremans et al., 2011).

The total diffusivity of the system is

$$
D=f D_{n}+(1-f) D_{e}
$$

where $f, D_{n}$ and $D_{e}$ represent the water fraction of the intra-neurite compartment, diffusivity of the intra- and extra-neurite compartment, respectively. Notice that the intrinsic intra-neurite diffusivity (i.e. the diffusivity along a cylinder axis) is $3 D_{n}$ for this model. Since macroscopic isotropy is assumed, the $3 \mathrm{D}$ and $6 \mathrm{D}$ diffusional kurtoses are equivalent to the 3D and 6D means of the diffusional kurtosis tensor, i.e. $\bar{W}=K$ and $\widetilde{W}=\widetilde{K}$, respectively. Given the fact that the 3D diffusional kurtosis for a compartment with uniformly oriented neurites is $12 / 5$ and that for a Gaussian compartment is vanishing, the total 3D diffusional kurtosis may be shown to be

$$
K=\bar{W}=3 f(1-f) \frac{\left(D_{e}-D_{n}\right)^{2}}{D^{2}}+f \frac{12}{5} \cdot \frac{D_{n}^{2}}{D^{2}}
$$

Because all the neurites are assumed to have the same intrinsic diffusivity (i.e., $3 D_{n}$ ), the $6 \mathrm{D}$ diffusional

kurtosis for the intra-neurite compartment is $\frac{5}{8} \cdot \frac{12}{5}=\frac{3}{2}$ (as follows from the lower bound in Eq. [13]). The total $6 \mathrm{D}$ diffusional kurtosis is then

$$
\widetilde{K}=\widetilde{W}=3 f(1-f) \frac{\left(D_{e}-D_{n}\right)^{2}}{D^{2}}+f \frac{3}{2} \cdot \frac{D_{n}^{2}}{D^{2}}
$$

By solving Eqs. [14] - [16], $f, D_{n}$ and $D_{e}$ as a function of $\delta \bar{W}$ are then found to be

$$
D_{n}=\frac{D}{9(\bar{W}-6 \delta \bar{W})}\{-30 \delta \bar{W} \pm \sqrt{30 \delta \bar{W}(3 \bar{W}-8 \delta \bar{W})(\bar{W}-6 \delta \bar{W}+3)}\}
$$




$$
\begin{aligned}
& f=\frac{10 D^{2} \delta \bar{W}}{9 D_{n}^{2}}, \\
& D_{e}=\frac{D-f D_{n}}{1-f} .
\end{aligned}
$$

Notice that due to the sign ambiguity in Eq. [17], there are generally two formal solutions for $D_{n}$. However, if one assumes that $0<D_{n}<D_{e}$, then the lower sign solution is excluded. The requirement $D_{n}<D_{e}$ is plausible, as water diffusion inside neurites is expected to be more restricted than for the extra-neurite compartment. The condition $0<D_{n}<D_{e}$ is satisfied for the upper sign solution provided $\bar{W}<12 / 5$, which holds for our data, and as long as Eq. [13] is valid. Therefore, only the upper sign solution in Eq. [17] will be considered for the purpose of GM modeling.

Figure 8 illustrates the ROI measurements of $f, D_{n}$ and $D_{e}$ in THA, PU and CAU for all subjects. The voxels that violated Eq. [13] were discarded from the ROI analysis, as these are inconsistent with an MGC model. The percentage of discarded voxels in THA, PU and CAU with respect to the ROIs used in Figure 4 for each subject were $(5,10,16,27) \%,(24,20,16,21) \%$ and $(37,30,25,17) \%$, respectively. These results indicate an intrinsic intra-neurite diffusivity of about $1.55 \pm 0.18 \mu \mathrm{m}^{2} / \mathrm{ms}$ in THA, $1.35 \pm 0.04 \mu \mathrm{m}^{2} / \mathrm{ms}$ in PU and $1.20 \pm 0.06 \mu \mathrm{m}^{2} / \mathrm{ms}$ in CAU. Since the free water diffusivity at $37{ }^{\circ} \mathrm{C}$ is about $3.0 \mu \mathrm{m}^{2} / \mathrm{ms}$ (Holz et al., 2000), we find a ratio for the intra-neurite and free diffusivities of about $0.52,0.45$ and 0.40 , for the three tissue types, respectively. These may be compared to the value of 0.46 for the ratio of the intra-neurite and free N-acetyl-L-aspartate (NAA) diffusivities obtained by Kroenke and coworkers (Kroenke et al., 2004). The intra-neurite water fractions were found to be $0.41 \pm 0.04$ in THA, $0.38 \pm 0.01$ in PU and $0.35 \pm 0.03$ in CAU. These are somewhat lower than histological values in mouse cortex of about 0.6 measured by Chklovskii and coworkers (Chklovskii et al., 2002). This disparity might reflect differences in brain regions or effects of intercompartmental water exchange not included in our model.

We emphasize that this model is highly idealized and neglects several aspects of diffusion in gray matter that may contribute to the dMRI signal. In addition to the aforementioned water exchange effect, these include non-Gaussianity and microscopic anisotropy in the extra-neurite compartment. Nonetheless, the model illustrates the potential of applying DP-DKI to help quantify brain microstructure. In particular, obtaining estimates for intrinsic intra-neurite diffusivity of water diffusion in gray matter has proven difficult with sPFG methods and is often fixed to an a priori value for modeling purposes (Hui et al., 2015; Zhang et al., 2012).

\section{Alternative methods for estimating microscopic anisotropy}

There are several alternative methods for estimating the type I microscopic anisotropy of brain tissues. In particular, those proposed by Lawrenz and Finsterbusch (Lawrenz and Finsterbusch , 2010), Jespersen and coworkers (Jespersen et al., 2014, 2013) and Szczepankiewicz and coworkers (Szczepankiewicz et al., 2014) 
have strong connections to the framework of DP-DKI, and the microscopic anisotropy metrics from these methods are closely related to $\delta \bar{W}$.

For example, Szczepankiewicz and coworkers (Szczepankiewicz et al., 2014), who consider only MGC models, define

$$
\mu \mathrm{FA} \equiv \sqrt{\frac{3}{2}}\left(1+\frac{\bar{D}^{2}}{\delta^{2} \lambda_{c}}\right)^{-\frac{1}{2}}
$$

With the help of Eq. [12], this can be rewritten as

$$
\mu \mathrm{FA}=\sqrt{\frac{3}{2}}\left(1+\frac{9 \bar{D}^{2}}{9 \delta^{2} \lambda+20 \bar{D}^{2} \delta \bar{W}}\right)^{-\frac{1}{2}} .
$$

Thus it is clear that $\delta \bar{W}$ and $\mu \mathrm{FA}$ contain essentially the same information for MGC models. As already discussed, the metric FE introduced by Jespersen and coworkers (Jespersen et al., 2014, 2013) is also equivalent to $\mu \mathrm{FA}$. Hence, these three, ostensibly distinct, diffusion MRI methods all lead to basically the same metric for microscopic anisotropy, at least for MGC models. It should be noted that, using the definition of FA (Basser and Pierpaoli, 1996), $\delta^{2} \lambda$ can be simply obtained from

$$
\delta^{2} \lambda=\frac{2 \bar{D}^{2} \mathrm{FA}^{2}}{3-2 \mathrm{FA}^{2}}
$$

Nonetheless, there are some key distinctions between the three approaches. First, both $\mu \mathrm{FA}$ and FE were introduced in the context of certain models, while $\delta \bar{W}$ is a pure diffusion metric without any necessary connection to specific model types. For MGC models, $\delta \bar{W}$ contains the same essential information on microscopic anisotropy as $\mu \mathrm{FA}$ and $\mathrm{FE}$, but it also has a more general meaning as a measure of non-Gaussian diffusion. Second, $\delta \bar{W}$ is an index, for MGC models, of type II microscopic anisotropy, and thus unlike type I metrics, it may vanish even when the FA is nonzero. Finally, the proposed data acquisition schemes are quite different. Szczepankiewicz and coworkers use a special magic-angle-spinning sequence, while Jespersen and coworkers recommend a d-PFG sequence with 60 different diffusion directions. Our 21 direction d-PFG protocol is arguably simpler than either of these. Moreover, both Szczepankiewicz and coworkers, and Jespersen and coworkers utilize powder averaging of the diffusion-weighted signal in their analyses, while powder averaging plays no role in our DP-DKI post-processing.

An essential difference between our conceptualization of type I and type II microscopic anisotropy is that type I microscopic anisotropy is necessarily nonzero in the presence of bulk anisotropy as reflected in conventional s-PFG metrics, such as the FA, while type II microscopic anisotropy is not. Operationally, we have thus defined type II microscopic anisotropy, as diffusion anisotropy that cannot be detected with s-PFG sequences, but requires d-PFG or higher order approaches. For MGC models, $\delta \bar{W}$ provides a natural metric of type II microscopic anisotropy and can only vanish if this property is absent. Nonetheless, the distinction between type I and type II microscopic anisotropy should not be overstated, since these concepts are closely related as indicated by Eq. [21]. Our intent is simply to help clarify the difference between two possible 
understandings of the term "microscopic anisotropy." For media without bulk anisotropy, type I and type II microscopic anisotropy are identical.

\section{Limitations and future studies}

A major limitation of the current study is a low SNR due to the relatively long TE (112 ms) despite the use of strong gradient sets, although our pulse sequence (Fig. 1) has a significantly shorter TE than alternative d-PFG pulse sequences in which each of the two diffusion gradient blocks includes a $180^{\circ} \mathrm{RF}$ pulse. The fact that there is a significant loss in SNR as compared to conventional DKI is a likely cause for a number of dark voids observed in the $\bar{W}$ and $\widetilde{W}$ maps (for example in the globus pallidus). These voids were largely absent when an additional DP-DKI experiment with twice the number of averages was performed (data not shown).

A second important limitation is that our protocol and analysis procedures did not incorporate methods for reducing the effects of background gradients and eddy currents, such as those discussed by Lawrenz and Finsterbusch (Lawrenz and Finsterbusch, 2011, 2013). The addition of such correction measures should improve the quality of the parametric maps derived with DP-DKI. Alternative approaches for reducing the influence of background gradients involve the use of modified pulse sequences (Shemesh and Cohen, 2011b). Nonetheless, the fact that there is very little angular modulation in d-PFG signal in a phantom experiment (data not shown) likely indicates that the effect of cross-term from imaging gradients may not be significant.

In future work, we will seek to compare the mean of the $6 \mathrm{D}$ tensors using the current method with that of full tensor estimations. An advantage of the latter is that constrained optimization can be employed to obtain biophysically plausible estimates that are more robust to noise, motion and imaging artifacts (Tabesh et al., 2011).

\section{Acknowledgement}

This work was supported by the Seed Funding Programme for Basic Research by The University of Hong Kong and by the Litwin Foundation.

\section{Appendix}

This appendix gives a derivation for Eq. [12], which relates $\delta \bar{W}$ to the variances for the compartmental 3D diffusion tensor eigenvalues.

From (Jensen et al., 2014), we have, for an MGC model with $N$ compartments,

$$
\widetilde{W}=\frac{5}{8} \bar{W}+\frac{9}{8 \bar{D}^{2}} \sum_{m=1}^{N} f_{m}\left[\bar{D}^{(m)}-\bar{D}\right]^{2}
$$


where $\bar{D}^{(m)}$ is the mean diffusivity for the $m$ th compartment and $f_{m}$ is the corresponding water fraction. The water fractions are normalized so that $\sum_{m=1}^{N} f_{m}=1$. Eq. [A1] can be rewritten to give

$$
\delta \bar{W}=\frac{3}{8} \bar{W}-\frac{9}{8 \bar{D}^{2}} \sum_{m=1}^{N} f_{m}\left[\bar{D}^{(m)}-\bar{D}\right]^{2} .
$$

Also from (Jensen et al., 2014), we have

$$
\bar{W}=\frac{3}{4 \pi \bar{D}^{2}} \int d \mathbf{\Omega} \sum_{m=1}^{N} f_{m}\left[D^{(m)}(\mathbf{n})-D(\mathbf{n})\right]^{2},
$$

where $D(\mathbf{n})$ is the diffusivity in a direction $\mathbf{n}$ for the full system and $D^{(m)}(\mathbf{n})$ is the diffusivity in a direction $\mathbf{n}$ for the $m$ th compartment. The integral is taken over all diffusion directions. By combining Eqs. [A.2] and [A.3], one sees that

$$
\delta \bar{W}=\frac{9}{32 \pi \bar{D}^{2}} \int d \mathbf{\Omega} \sum_{m=1}^{N} f_{m}\left\{\left[D^{(m)}(\mathbf{n})-D(\mathbf{n})\right]^{2}-\left[\bar{D}^{(m)}-\bar{D}\right]^{2}\right\},
$$

which may be rearranged to

$$
\delta \bar{W}=\frac{9}{32 \pi \bar{D}^{2}} \int d \mathbf{\Omega} \sum_{m=1}^{N} f_{m}\left\{\left[D^{(m)}(\mathbf{n})\right]^{2}-[D(\mathbf{n})]^{2}-\left[\bar{D}^{(m)}\right]^{2}+\bar{D}^{2}\right\} .
$$

After performing the angular integrals, this becomes

$$
\delta \bar{W}=\frac{9}{8 \bar{D}^{2}} \sum_{m=1}^{N} f_{m}\left\{\bar{D}^{2}-\left[\bar{D}^{(m)}\right]^{2}+\frac{1}{15} \sum_{i, j=1}^{3}\left[D_{i i}^{(m)} D_{j j}^{(m)}+2 D_{i j}^{(m)} D_{i j}^{(m)}-D_{i i} D_{j j}-2 D_{i j} D_{i j}\right]\right\} .
$$

with $D_{i j}$ being a component of $\mathbf{D}$ and $D_{i j}^{(m)}$ being a component of the diffusion tensor $\mathbf{D}^{(m)}$ for the $m$ th compartment. By using the facts that

$$
\bar{D}=\frac{1}{3} \sum_{i=1}^{3} D_{i i}
$$

and

$$
\bar{D}^{(m)}=\frac{1}{3} \sum_{i=1}^{3} D_{i i}^{(m)},
$$

one may verify that Eq. [A.6] is equivalent to

$$
\delta \bar{W}=\frac{9}{8 \bar{D}^{2}} \sum_{m=1}^{N} f_{m}\left\{\frac{2}{5} \bar{D}^{2}-\frac{2}{5}\left[\bar{D}^{(m)}\right]^{2}+\frac{2}{15} \sum_{i, j=1}^{3}\left[D_{i j}^{(m)} D_{i j}^{(m)}-D_{i j} D_{i j}\right]\right\} .
$$

In terms of the diffusion tensor eigenvalues, this takes the form

$$
\delta \bar{W}=\frac{9}{20 \bar{D}^{2}} \sum_{m=1}^{N} f_{m}\left\{\bar{D}^{2}-\left[\bar{D}^{(m)}\right]^{2}+\frac{1}{3} \sum_{i=1}^{3}\left[\left(\lambda_{i}^{(m)}\right)^{2}-\left(\lambda_{i}\right)^{2}\right]\right\},
$$

where $\lambda_{i}^{(m)}$ is the $i$ th eigenvalue of $\mathbf{D}^{(m)}$ and $\lambda_{i}$ is the $i$ th eigenvalue of $\mathbf{D}$. By applying the definitions

$$
\delta^{2} \lambda \equiv \frac{1}{3} \sum_{i=1}^{3}\left[\left(\lambda_{i}\right)^{2}-\bar{D}^{2}\right]
$$

for the variance of the total diffusion tensor eigenvalues and

$$
\delta^{2} \lambda_{c} \equiv \frac{1}{3} \sum_{m=1}^{N} f_{m} \sum_{i=1}^{3}\left[\left(\lambda_{i}^{(m)}\right)^{2}-\left(\bar{D}^{(m)}\right)^{2}\right]
$$


for the variance of the compartmental diffusion tensor eigenvalues to Eq. [A.10], we finally arrive at Eq. [12]. 


\section{References}

Ackerman, J.J.H., Neil, J.J., 2010. The use of MR-detectable reporter molecules and ions to evaluate diffusion in normal and ischemic brain. NMR Biomed. doi:10.1002/nbm.1530

Alexander, A.L., Hasan, K.M., Lazar, M., Tsuruda, J.S., Parker, D.L., 2001. Analysis of partial volume effects in diffusion-tensor MRI. Magn. Reson. Med. 45, 770-780. doi:10.1002/mrm.1105

Basser, P.J., Pierpaoli, C., 1996. Microstructural and physiological features of tissues elucidated by quantitativediffusion-tensor MRI. J. Magn. Reson. B 111, 209-219. doi:10.1016/j.jmr.2011.09.022

Callaghan, P.T., Komlosh, M.E., 2002. Locally anisotropic motion in a macroscopically isotropic system: displacement correlations measured using double pulsed gradient spin-echo NMR. Magn. Reson. Chem. 40, S15-S19. doi: $10.1002 / \mathrm{mrc} .1122$

Chklovskii, D.B., Schikorski, T., Stevens, C.F., 2002. Wiring optimization in cortical circuits. Neuron. doi:10.1016/S0896-6273(02)00679-7

De Santis, S., Drakesmith, M., Bells, S., Assaf, Y., Jones, D.K., 2014. Why diffusion tensor MRI does well only some of the time: Variance and covariance of white matter tissue microstructure attributes in the living human brain. Neuroimage 89, 35-44. doi:10.1016/j.neuroimage.2013.12.003

Feinberg, D.A., Setsompop, K., 2013. Ultra-fast MRI of the human brain with simultaneous multi-slice imaging. J. Magn. Reson. 229, 90-100. doi:10.1016/j.jmr.2013.02.002

Hansen, B., Lund, T.E., Sangill, R., Jespersen, S.N., 2013. Experimentally and computationally fast method for estimation of a mean kurtosis. Magn. Reson. Med. 69, 1754-60. doi:10.1002/mrm.25090

Hansen, B., Lund, T.E., Sangill, R., Jespersen, S.N., 2014. Erratum: Hansen, Lund, Sangill, and Jespersen. Experimentally and Computationally Fast Method for Estimation of a Mean Kurtosis. Magnetic Resonance in Medicine 69:1754-1760 (2013). Magn. Reson. Med. 71, 2250. doi:10.1002/mrm.25090

Holz, M., Heil, S.R., Sacco, A., 2000. Temperature-dependent self-diffusion coefficients of water and six selected molecular liquids for calibration in accurate 1H NMR PFG measurements. Phys. Chem. Chem. Phys. doi:10.1039/b005319h

Hui, E.S., Glenn, G.R., Helpern, J.A., Jensen, J.H., 2015. NeuroImage Kurtosis analysis of neural diffusion organization. Neuroimage 42, 122-134. doi:10.1016/j.neuroimage.2014.11.015

Jensen, J.H., 2014. Sufficiency of diffusion tensor in characterizing the diffusion MRI signal to leading order in diffusion weighting. NMR Biomed. 27, 1005-1007. doi:10.1002/nbm.3145

Jensen, J.H., Helpern, J.A., 2011. Effect of gradient pulse duration on MRI estimation of the diffusional kurtosis for a two-compartment exchange model. J. Magn. Reson. 210, 233-237. doi:10.1016/j.jmr.2011.03.012

Jensen, J.H., Hui, E.S., Helpern, J.A., 2014. Double-pulsed diffusional kurtosis imaging. NMR Biomed. 27, 363-370. doi:10.1002/nbm.3094 
Jespersen, S.N., 2012. Equivalence of double and single wave vector diffusion contrast at low diffusion weighting. NMR Biomed. 25, 813-8. doi:10.1002/nbm.1808

Jespersen, S.N., Lundell, H., Sønderby, C.K., Dyrby, T.B., 2013. Orientationally invariant metrics of apparent compartment eccentricity from double pulsed field gradient diffusion experiments. NMR Biomed. 26, 1647-62. doi:10.1002/nbm.2999

Jespersen, S.N., Lundell, H., Sønderby, C.K., Dyrby, T.B., 2014. Erratum: Orientationally invariant metrics of apparent compartment eccentricity from double pulsed field gradient diffusion experiments. NMR Biomed. 27, 738-738. doi: $10.1002 / \mathrm{nbm} .3125$

Komlosh, M.E., Horkay, F., Freidlin, R.Z., Nevo, U., Assaf, Y., Basser, P.J., 2007. Detection of microscopic anisotropy in gray matter and in a novel tissue phantom using double Pulsed Gradient Spin Echo MR. J. Magn. Reson. 189, 38-45. doi:10.1016/j.jmr.2007.07.003

Komlosh, M.E., Lizak, M.J., Horkay, F., Freidlin, R.Z., Basser, P.J., 2008. Observation of microscopic diffusion anisotropy in the spinal cord using double-pulsed gradient spin echo MRI. Magn. Reson. Med. 59, 803-9. doi:10.1002/mrm.21528

Kroenke, C.D., Ackerman, J.J.H., Yablonskiy, D.A., 2004. On the nature of the NAA diffusion attenuated MR signal in the central nervous system. Magn. Reson. Med. 52, 1052-1059. doi:10.1002/mrm.20260

Lasič, S., Szczepankiewicz, F., Eriksson, S., Nilsson, M., Topgaard, D., 2014. Microanisotropy imaging: quantification of microscopic diffusion anisotropy and orientational order parameter by diffusion MRI with magic-angle spinning of the q-vector. Front. Phys. 2, 1-14. doi:10.3389/fphy.2014.00011

Lawrenz, M., Finsterbusch, J., 2011. Detection of microscopic diffusion anisotropy on a whole-body MR system with double wave vector imaging. Magn. Reson. Med. 66, 1405-15. doi:10.1002/mrm.22934

Lawrenz, M., Finsterbusch, J., 2013. Double-wave-vector diffusion-weighted imaging reveals microscopic diffusion anisotropy in the living human brain. Magn. Reson. Med. 69, 1072-82. doi:10.1002/mrm.24347

Lawrenz, M., Finsterbusch, J., 2014. Mapping measures of microscopic diffusion anisotropy in human brain white matter in vivo with double-wave-vector diffusion-weighted imaging. Magn. Reson. Med. 73, 773-83. doi:10.1002/mrm.25140

Lawrenz, M., Koch, M. a, Finsterbusch, J., 2010. A tensor model and measures of microscopic anisotropy for doublewave-vector diffusion-weighting experiments with long mixing times. J. Magn. Reson. 202, 43-56. doi:10.1016/j.jmr.2009.09.015

Mitra, P.P., 1995. Multiple wave-vector extensions of the NMR pulsed-field-gradient spin-echo diffusion measurement. Phys. Rev. B 51, 15074-15078. doi:10.1103/PhysRevB.51.15074

Nilsson, M., Lätt, J., Van Westen, D., Brockstedt, S., Lasič, S., Ståhlberg, F., Topgaard, D., 2013. Noninvasive mapping of water diffusional exchange in the human brain using filter-exchange imaging. Magn. Reson. Med. 69, 15731581. doi: $10.1002 / \mathrm{mrm} .24395$

Ozarslan, E., 2009. Compartment shape anisotropy (CSA) revealed by double pulsed field gradient MR. J. Magn. Reson. 199, 56-67. doi:10.1016/j.jmr.2009.04.002

Ozarslan, E., Basser, P.J., 2008. Microscopic anisotropy revealed by NMR double pulsed field gradient experiments with arbitrary timing parameters. J. Chem. Phys. 128, 154511. doi:10.1063/1.2905765 
Panagiotaki, E., Schneider, T., Siow, B., Hall, M.G., Lythgoe, M.F., Alexander, D.C., 2012. Compartment models of the diffusion MR signal in brain white matter: A taxonomy and comparison. Neuroimage 59, 2241-2254.

doi:10.1016/j.neuroimage.2011.09.081

Shemesh, N., Barazany, D., Sadan, O., Bar, L., Zur, Y., Barhum, Y., Sochen, N., Offen, D., Assaf, Y., Cohen, Y., 2012a. Mapping apparent eccentricity and residual ensemble anisotropy in the gray matter using angular double-pulsedfield-gradient MRI. Magn. Reson. Med. 68, 794-806. doi:10.1002/mrm.23300

Shemesh, N., Cohen, Y., 2011a. Microscopic and compartment shape anisotropies in gray and white matter revealed by angular bipolar double-PFG MR. Magn. Reson. Med. 65, 1216-27. doi:10.1002/mrm.22738

Shemesh, N., Cohen, Y., 2011b. Overcoming apparent Susceptibility-Induced Anisotropy (aSIA) by bipolar doublePulsed-Field-Gradient NMR. J. Magn. Reson. 212, 362-369. doi:10.1016/j.jmr.2011.07.015

Shemesh, N., Özarslan, E., Adiri, T., Basser, P.J., Cohen, Y., 2010a. Noninvasive bipolar double-pulsed-field-gradient NMR reveals signatures for pore size and shape in polydisperse, randomly oriented, inhomogeneous porous media. J. Chem. Phys. 133. doi:10.1063/1.3454131

Shemesh, N., Özarslan, E., Basser, P.J., Cohen, Y., 2012b. Accurate noninvasive measurement of cell size and compartment shape anisotropy in yeast cells using double-pulsed field gradient MR. NMR Biomed. 25, 236-46. doi:10.1002/nbm.1737

Shemesh, N., Ozarslan, E., Komlosh, M.E., Basser, P.J., Cohen, Y., 2010b. From single-pulsed field gradient to doublepulsed field gradient MR: gleaning new microstructural information and developing new forms of contrast in MRI. NMR Biomed. 23, 757-80. doi:10.1002/nbm.1550

Szczepankiewicz, F., Lasič, S., van Westen, D., Sundgren, P.C., Englund, E., Westin, C.-F., Ståhlberg, F., Lätt, J., Topgaard, D., Nilsson, M., 2014. Quantification of microscopic diffusion anisotropy disentangles effects of orientation dispersion from microstructure: Applications in healthy volunteers and in brain tumors. Neuroimage 104, 241-252. doi:10.1016/j.neuroimage.2014.09.057

Tabesh, A., Jensen, J.H., Ardekani, B.A., Helpern, J.A., 2011. Estimation of tensors and tensor-derived measures in diffusional kurtosis imaging. Magn. Reson. Med. 65, 823-36. doi:10.1002/mrm.22655

Zhang, H., Schneider, T., Wheeler-Kingshott, C.A., Alexander, D.C., 2012. NODDI: Practical in vivo neurite orientation dispersion and density imaging of the human brain. Neuroimage 61, 1000-1016.

doi:10.1016/j.neuroimage.2012.03.072 


\section{Figure Captions}

1. The pulse sequence diagram of the current d-PFG implementation using a spin-echo echo-planar imaging sequence, consisting of a first block of diffusion gradients applied before the $180^{\circ} \mathrm{RF}$ pulse and a second block of diffusion gradients applied after the $180^{\circ} \mathrm{RF}$ pulse. The diffusion gradient strength $g$, duration $\delta$ and diffusion time $\Delta$ of both blocks are set to be identical. A mixing time $\tau$ separates the end of the first block and the beginning of the second block. The first and second blocks are applied along the phase encoding (PE) and frequency encoding (FE) directions, respectively.

2. Plot of $\tilde{\psi}$ (square) and $\psi$ (circle) versus $\tilde{b}$ as measured from the normalized d-PFG signal $\tilde{S}(\tilde{b}, \widetilde{\mathbf{n}}) / \tilde{S}_{0}$ in the corpus callosum (CC), corticospinal tract (CT), thalamus (THA), putamen (PU) and caudate nucleus (CAU) of a representative volunteer. Data points are expressed as the mean and standard deviation of all values in the corresponding ROI. The fits for $\tilde{\psi}$ to Eq. [10] (solid line) and for $\psi$ to Eq. [11] (dashed line) are also shown. The ROI definitions for each brain structure are shown in the inset of each signal plot.

3. Angular dependence of d-PFG signal at b-value of $2000 \mathrm{~s} / \mathrm{mm}^{2}$ expressed as percent change in signal deviation. $\theta$ is the angle between the diffusion wave vector of the first and second blocks of PFG. $\bar{S}$ is the average of d-PFG signals acquired with the direction of the first diffusion wave vector being fixed while that of the second wave vector varied. The current diffusion-encoding scheme in Table 1 resulted in four different signal curves. It is evident that WM structures (CC and CT) have the largest angular modulations, whilst GM structures (PU and CAU) have the smallest.

4. $\bar{D}, \widetilde{\bar{D}},|\bar{D}-\widetilde{\bar{D}}|, \bar{W}, \widetilde{W}$ and $\delta \bar{W}$ measurements for all four subjects. The measurements are expressed as mean and standard deviation of all values in the corresponding ROI for each subject. Paired t-tests were performed to compare $\widetilde{D}$ and $\widetilde{\bar{D}}$ as well as $\bar{W}$ and $\widetilde{W}$ for all ROIs and all subjects. * p-value < 0.01. There are a number of significant differences between the two metrics, despite the small absolute difference between $\bar{D}$ and $\widetilde{\bar{D}}$ (maximum absolute difference of only $0.07 \pm 0.02 \mu \mathrm{m}^{2} / \mathrm{ms}$ ). Again, similar to Figures 2 and 3, WM structures have the largest $\delta \bar{W}$, and GM structures have the smallest. The difference between $\bar{W}$ and $\widetilde{W}$ was not significant for the CAU of subjects 1 to 3 .

5. $\bar{D}, \widetilde{\bar{D}},|\bar{D}-\widetilde{\bar{D}}|, \bar{W}, \widetilde{W}, \delta \bar{W}$ and FA maps of a representative subject. Note that the FA maps were computed from the DP-DKI data acquired with the first 9 diffusion-encoding directions. The calibration bars for $\bar{D}, \widetilde{\bar{D}}$, and $|\bar{D}-\widetilde{\bar{D}}|$ are in units of $\mu \mathrm{m}^{2} / \mathrm{ms}$, while the other calibration bars are dimensionless. Notice the difference in the contrast of WM between $\delta \bar{W}$ and FA. 
6. The $\widetilde{W}$ and $\delta \bar{W}$ maps of all subjects for illustration of inter-subject variability. Despite of the dark voids in $\widetilde{W}$, the maps amongst all subjects are fairly consistent. The voids were likely due to insufficient signal-to-noise ratio.

7. Scatter plot for $\widetilde{W}$ (left) and $\delta \bar{W}$ (right) against $\bar{W}$ for various brain structures of all subjects. For each ROI in the plots, pixels from individual subjects are grouped together. The total number of pixels for CC, CT, THA, PU and CAU are 230, 335, 343, 121, and 105, respectively. The Spearman correlation coefficient of $\widetilde{W}$ for WM structures is 0.72 , for THA is 0.75 , and for GM structures is 0.79 . Whereas that of $\delta W$ for WM structures is 0.27 , for THA is 0.48 , and for GM structures is 0.34 .

8. $f, D_{n}$ and $D_{e}$ estimates for all four subjects using the GM model of Eqs. [17]-[19]. The estimates are expressed as mean and standard deviation of all values in the corresponding ROI for each subject. The voxels that violated Eq. [13] were discarded from the ROI analysis. The intrinsic intra-neurite diffusivity for THA, PU and CAU are $1.55 \pm 0.18,1.35 \pm 0.04$ and $1.20 \pm 0.06 \mu \mathrm{m}^{2} / \mathrm{ms}$, respectively. The intra-neurite water fractions are $0.41 \pm 0.04,0.38 \pm 0.01$ and $0.35 \pm 0.03$, respectively. 
Table 1. 6D diffusion-encoding directions.

\begin{tabular}{|c|c|}
\hline$m$ & $\widetilde{\mathbf{n}}_{m}$ \\
\hline $\mathbf{1}$ & $(1,0,0,0,0,0)$ \\
\hline $\mathbf{2}$ & $(0,1,0,0,0,0)$ \\
\hline $\mathbf{3}$ & $(0,0,1,0,0,0)$ \\
\hline $\mathbf{4}$ & $(1,1,0,0,0,0) / \sqrt{2}$ \\
\hline $\mathbf{5}$ & $(1,-1,0,0,0,0) / \sqrt{2}$ \\
\hline $\mathbf{6}$ & $(1,0,1,0,0,0) / \sqrt{2}$ \\
\hline $\mathbf{7}$ & $(1,0,-1,0,0,0) / \sqrt{2}$ \\
\hline $\mathbf{8}$ & $(0,1,1,0,0,0) / \sqrt{2}$ \\
\hline $\mathbf{9}$ & $(0,1,-1,0,0,0) / \sqrt{2}$ \\
\hline $\mathbf{1 0}$ & $(1,0,0,0,1,0) / \sqrt{2}$ \\
\hline $\mathbf{1 1}$ & $(1,0,0,0,-1,0) / \sqrt{2}$ \\
\hline $\mathbf{1 2}$ & $(1,0,0,0,0,1) / \sqrt{2}$ \\
\hline $\mathbf{1 3}$ & $(1,0,0,0,0,-1) / \sqrt{2}$ \\
\hline $\mathbf{1 4}$ & $(0,1,0,0,0,1) / \sqrt{2}$ \\
\hline $\mathbf{1 5}$ & $(0,1,0,0,0,-1) / \sqrt{2}$ \\
\hline $\mathbf{1 6}$ & $(1,0,0,1,0,0) / \sqrt{2}$ \\
\hline $\mathbf{1 7}$ & $(1,0,0,-1,0,0) / \sqrt{2}$ \\
\hline $\mathbf{1 8}$ & $(0,1,0,0,1,0) / \sqrt{2}$ \\
\hline $\mathbf{1 9}$ & $(0,1,0,0,-1,0) / \sqrt{2}$ \\
\hline $\mathbf{2 0}$ & $(0,0,1,0,0,1) / \sqrt{2}$ \\
\hline $\mathbf{2 1}$ & $(0,0,1,0,0,-1) / \sqrt{2}$ \\
\hline
\end{tabular}




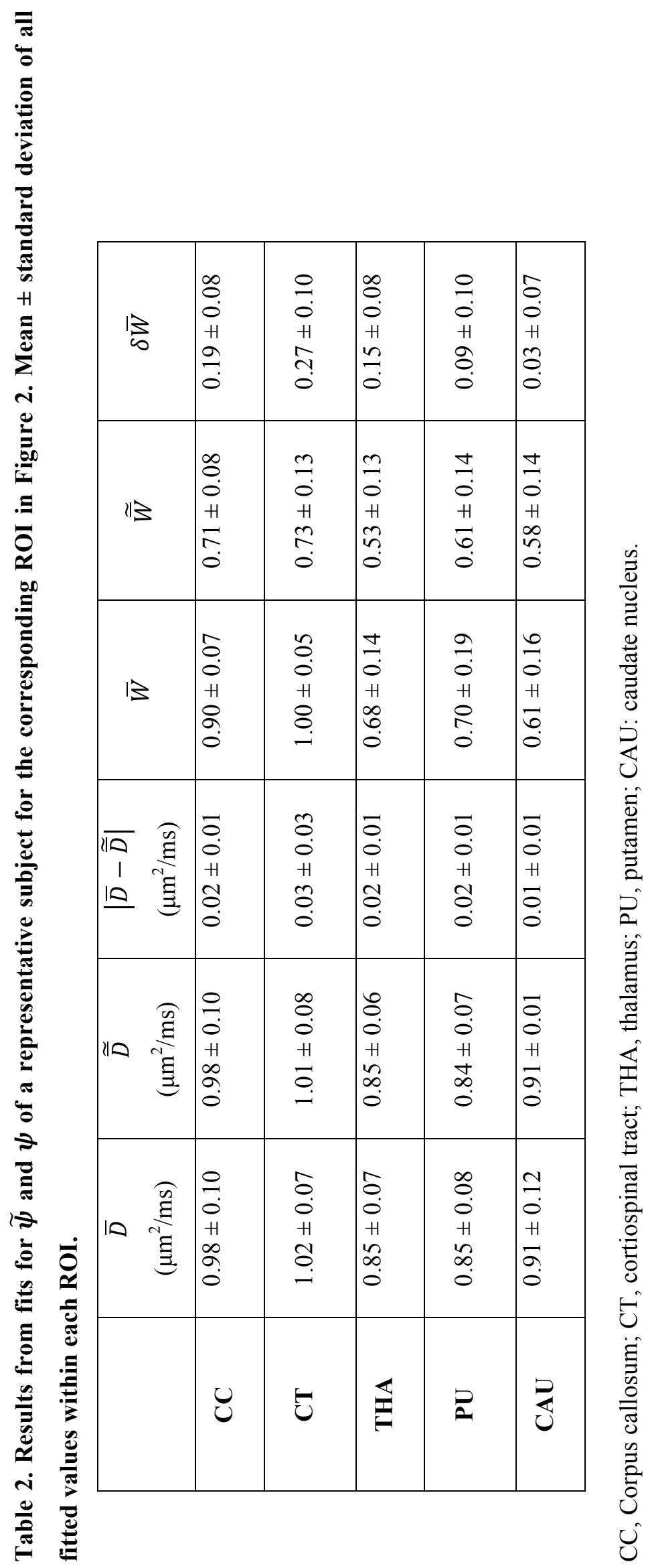




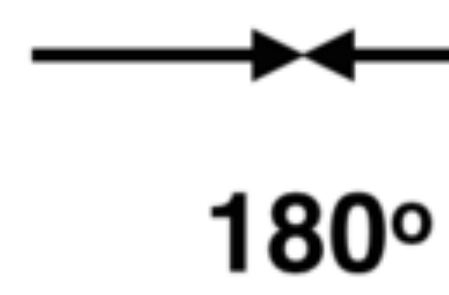

\section{$90^{\circ}$}

RF

FE

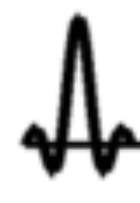

PE

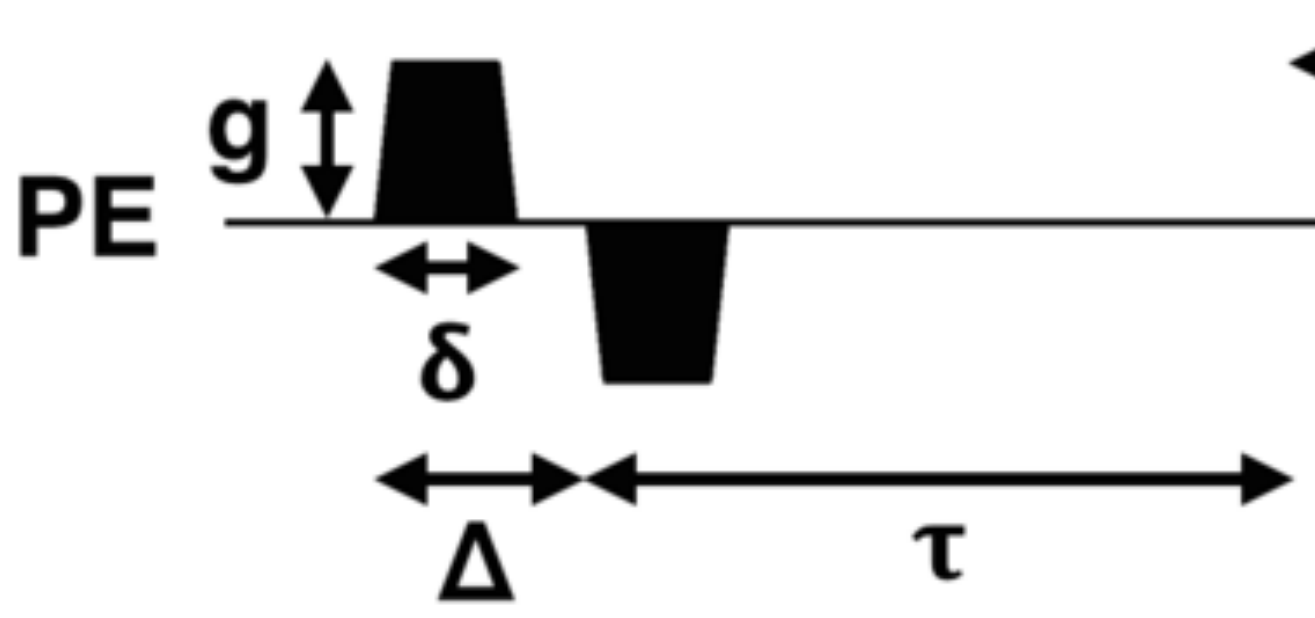

\section{echo}
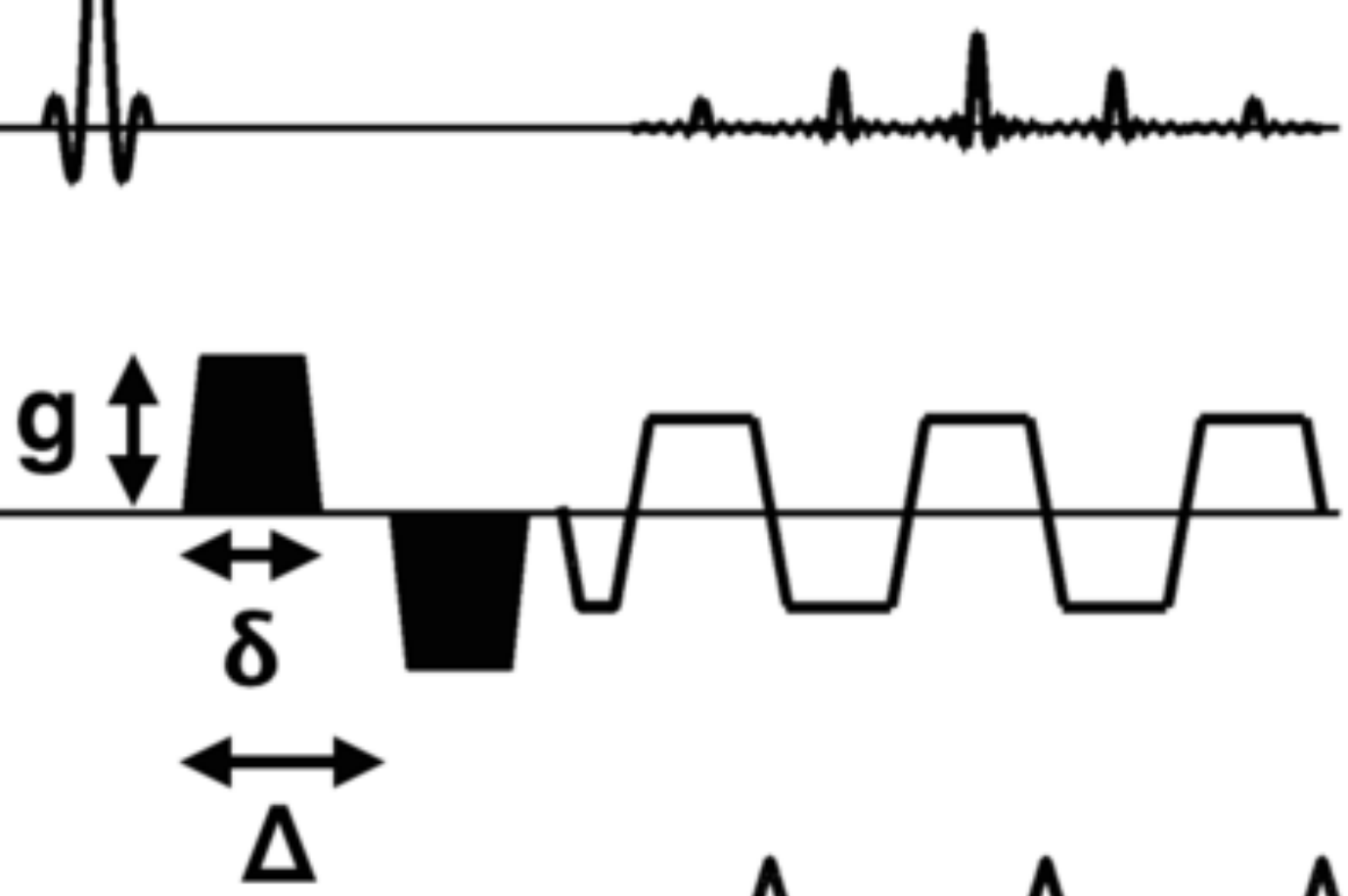

$\Lambda \quad \Lambda$

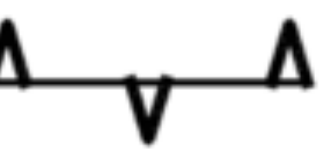


Click here to download high resolution image
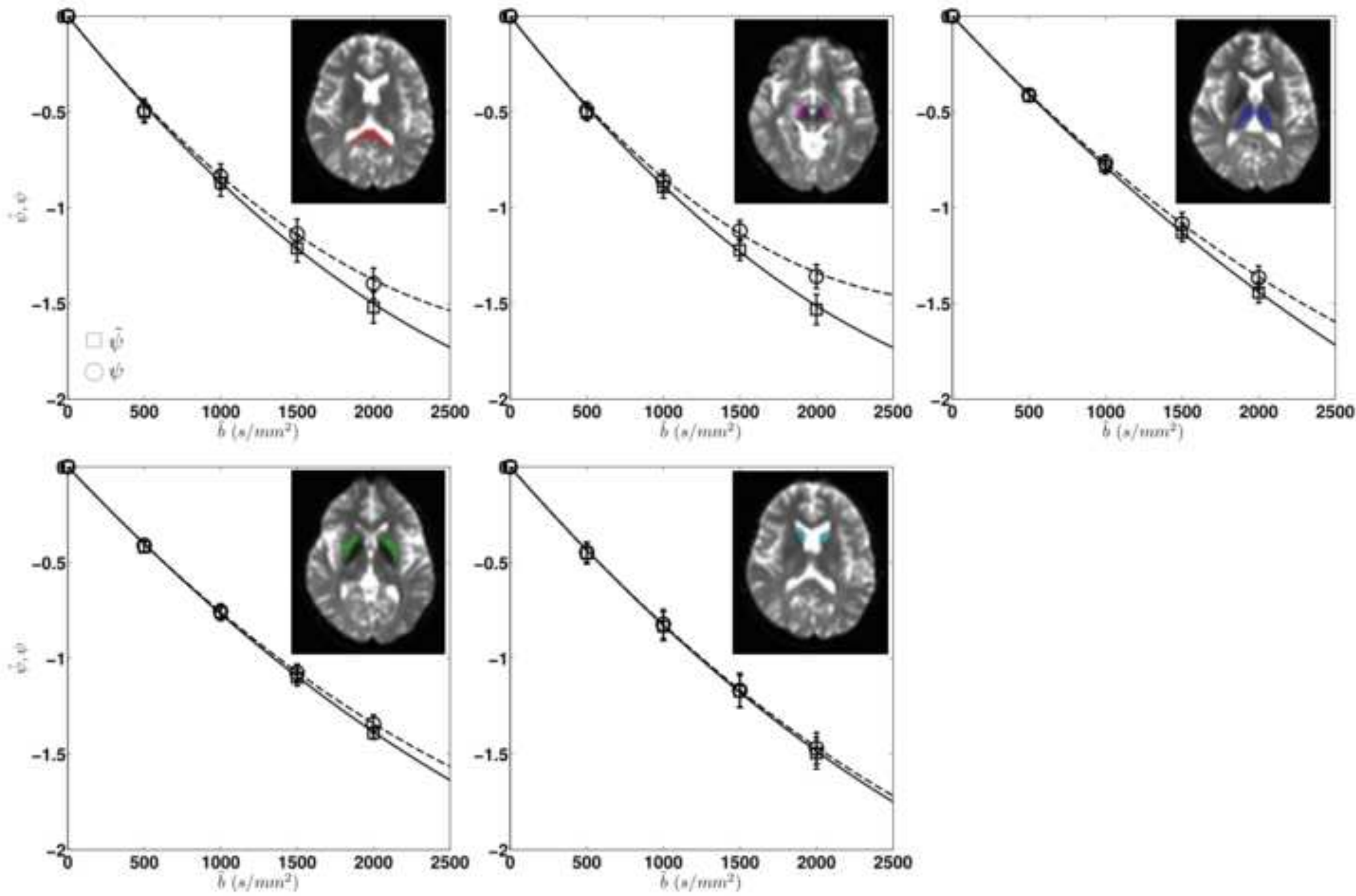


\section{Figure 3}

Click here to download high resolution image
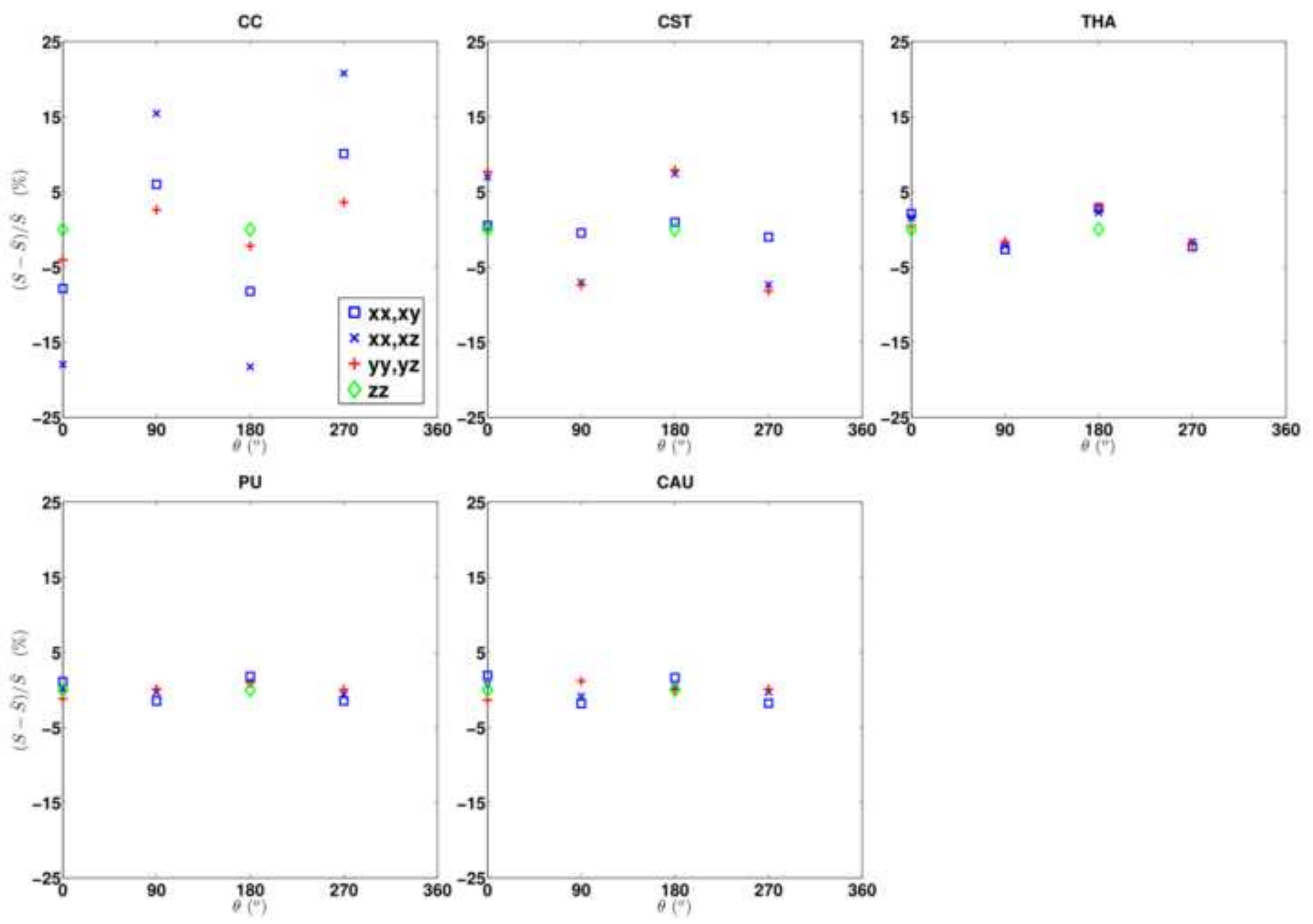

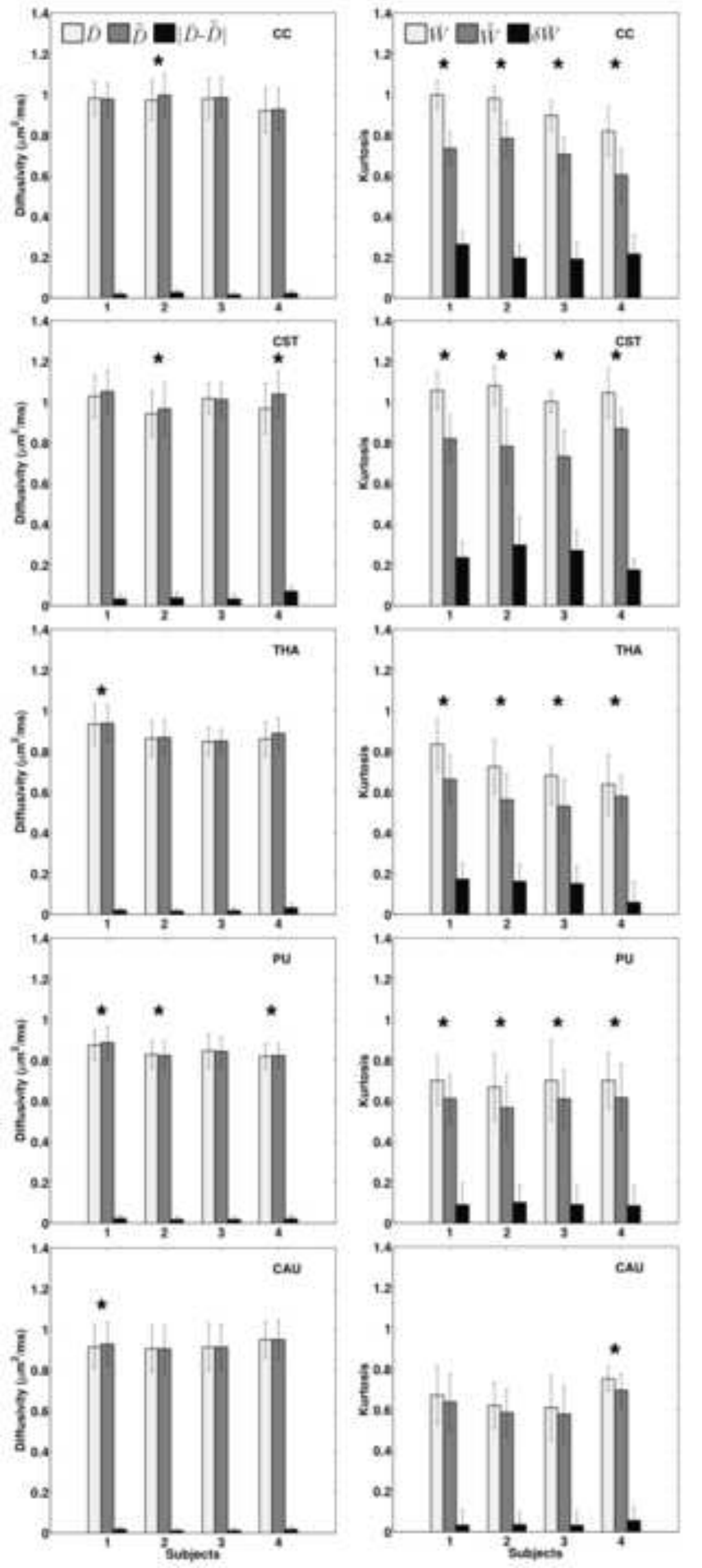


\section{Figure 5}

Click here to download high resolution image

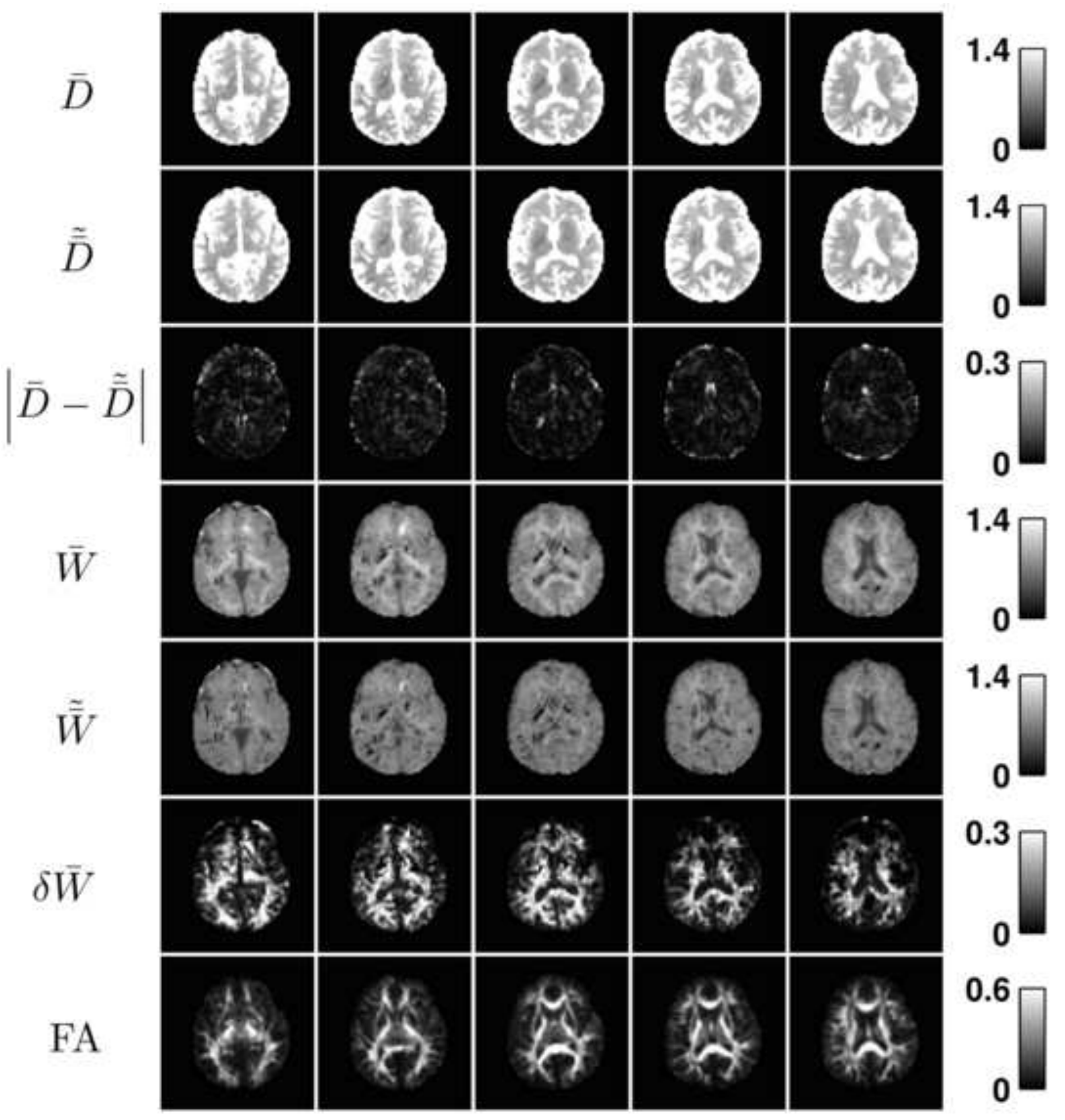



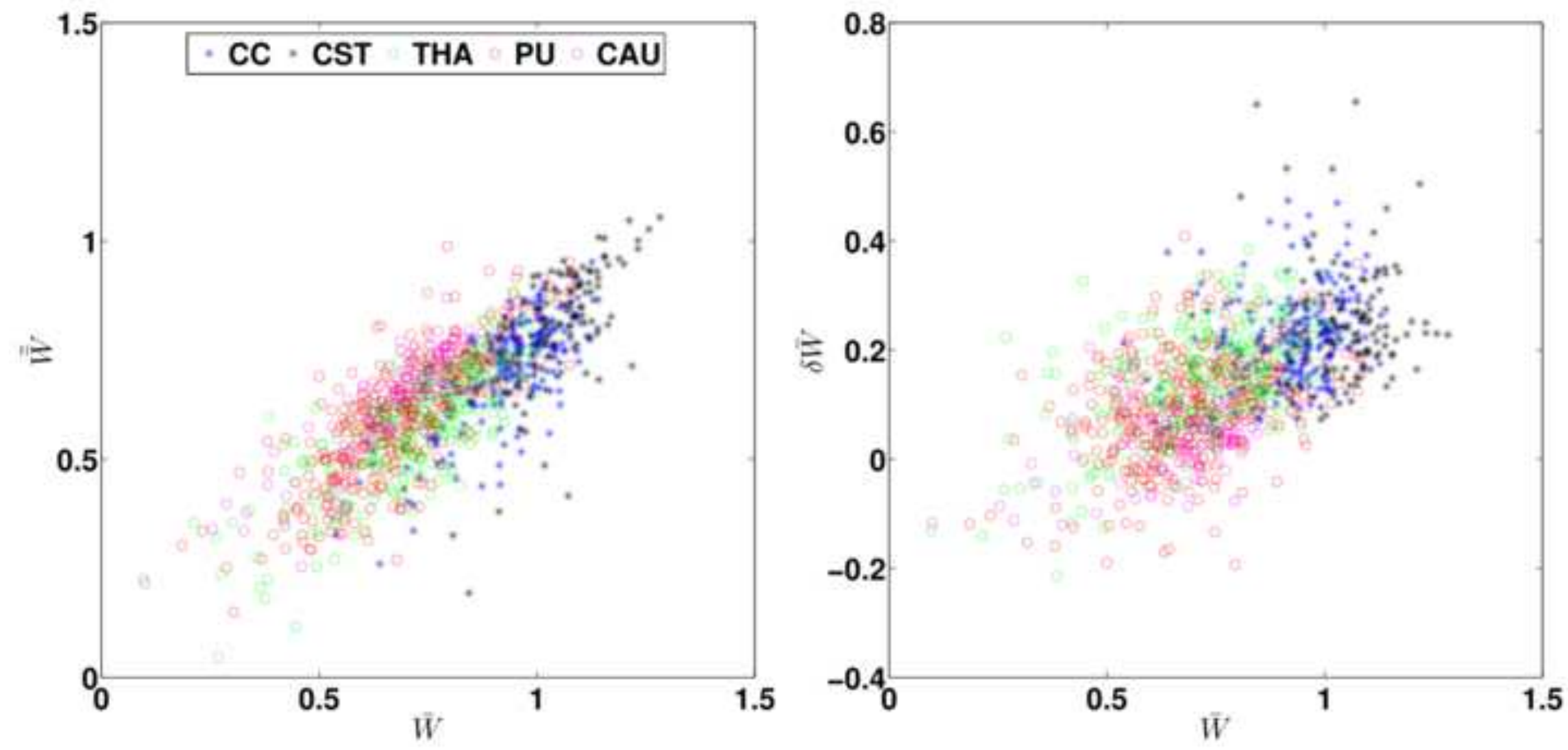


\section{Figure 8}

Click here to download high resolution image
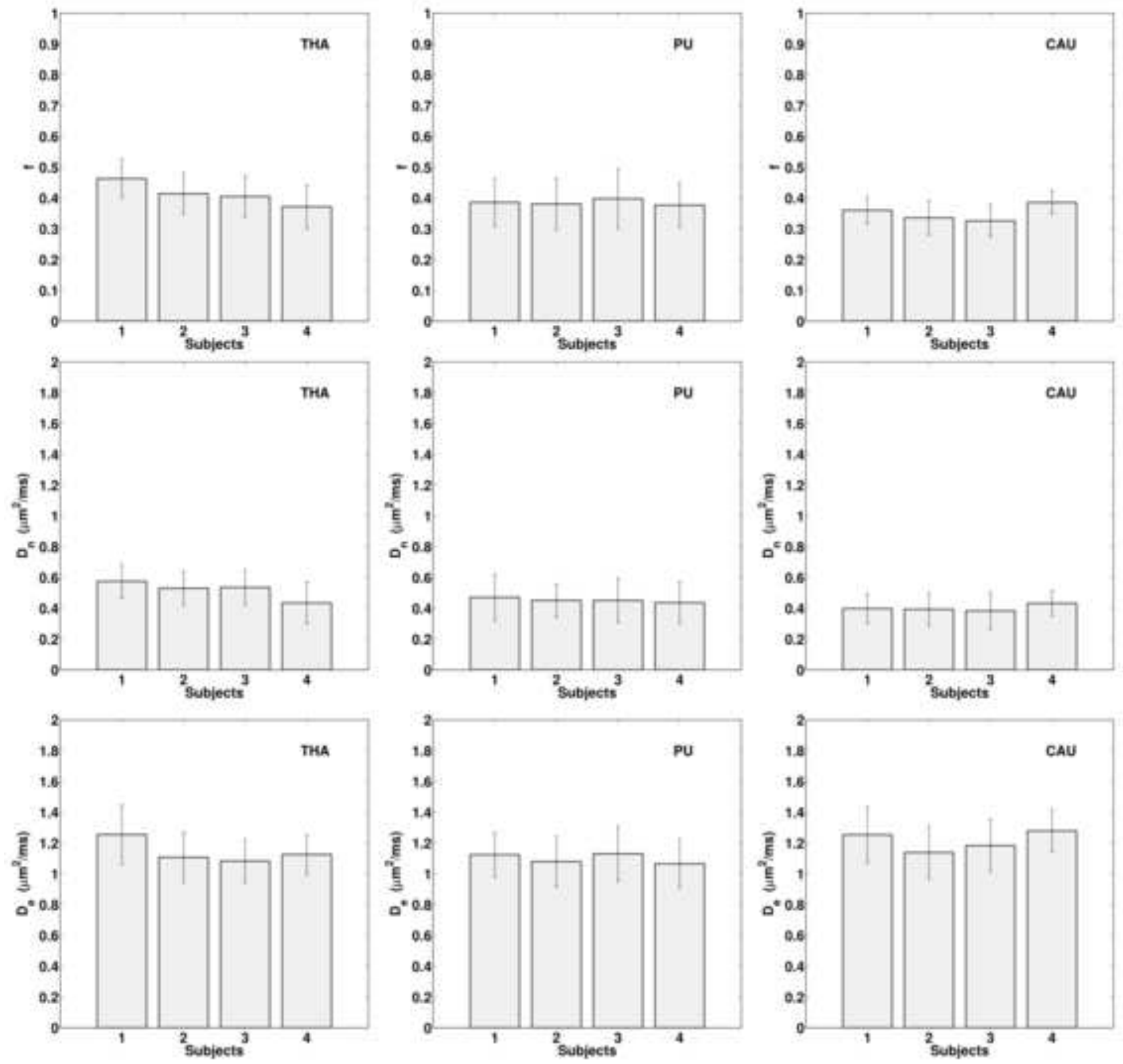